\title{
Unraveling Two-Photon Entanglement via the Squeezing Spectrum of Light Traveling through Nanofiber-Coupled Atoms
}

\author{
Jakob Hinney, ${ }^{1, \dagger}$ Adarsh S. Prasad, ${ }^{1}$ Sahand Mahmoodian $\odot,{ }^{2,, *}$ Klemens Hammerer®, ${ }^{2}$ Arno Rauschenbeutel, ${ }^{1,3}$ \\ Philipp Schneeweiss $\mathbb{B}^{1,3}$ Jürgen Volz, ${ }^{1,3}$ and Max Schemmer® ${ }^{3,8}$ \\ ${ }^{1}$ Vienna Center for Quantum Science and Technology, TU Wien-Atominstitut, Stadionallee 2, 1020 Vienna, Austria \\ ${ }^{2}$ Institute for Theoretical Physics, Institute for Gravitational Physics (Albert Einstein Institute), Leibniz University Hannover, \\ Appelstraße 2, 30167 Hannover, Germany \\ ${ }^{3}$ Department of Physics, Humboldt-Universität zu Berlin, 10099 Berlin, Germany
}

(Received 20 November 2020; accepted 22 July 2021; published 14 September 2021)

\begin{abstract}
We observe that a weak guided light field transmitted through an ensemble of atoms coupled to an optical nanofiber exhibits quadrature squeezing. From the measured squeezing spectrum we gain direct access to the phase and amplitude of the energy-time entangled part of the two-photon wave function which arises from the strongly correlated transport of photons through the ensemble. For small atomic ensembles we observe a spectrum close to the line shape of the atomic transition, while sidebands are observed for sufficiently large ensembles, in agreement with our theoretical predictions. Furthermore, we vary the detuning of the probe light with respect to the atomic resonance and infer the phase of the entangled twophoton wave function. From the amplitude and the phase of the spectrum, we reconstruct the real and imaginary part of the time-domain wave function. Our characterization of the entangled two-photon component constitutes a diagnostic tool for quantum optics devices.
\end{abstract}

DOI: 10.1103/PhysRevLett.127.123602

Nonclassical states of light are at the heart of quantum optics. Many experimental approaches for the generation of nonclassical states of light are based on strong coupling between photons and quantum emitters, e.g., making use of resonant enhancement of atom-light interaction via high finesse optical cavities [1-8] or employing collective response of strongly interacting Rydberg atoms [9-16]. These approaches aim to maximize the interaction strength between atoms and photons to generate nonclassical states of light.

In contrast, it has recently been predicted that light with nonclassical signatures can be generated in a conceptually simple system consisting of $N$ two-level emitters weakly coupled to a continuum of modes propagating in a onedimensional waveguide and driven with a coherent laser field [17]. This occurs even when the atoms are driven far below saturation. This has led to the observation of highly correlated states of light which can be tuned to exhibit antibunching or bunching by controlling the optical depth of the atomic ensemble [18]. At low input powers, the photon correlations arise from the two-photon component of the field, which can be written as a superposition of a

Published by the American Physical Society under the terms of the Creative Commons Attribution 4.0 International license. Further distribution of this work must maintain attribution to the author(s) and the published article's title, journal citation, and DOI. separable part and an entangled part [19]. Measuring the second-order correlation function of the field, as in [18], probes the absolute magnitude of the entire two-photon component. However, it does not provide direct access to the relative phase and amplitude of the entangled and separable parts.

Here, we report the observation of quadrature squeezing [20-24] of the light that is transmitted through an ensemble of atoms coupled to a nanofiber. While the measurement of squeezing at low powers in a weakly coupled atomic ensemble is novel in itself, we also use this measurement to gain direct experimental access to the phase and magnitude of the entangled part of the transmitted field. More precisely, to leading order, the squeezing spectrum is proportional to the amplitude of the entangled part of the two-photon wave function, and the homodyne measurement of a continuous field allows us to measure the entanglement of the spectral components of opposite frequency, $\omega_{0} \pm \omega$, around the probe field with frequency $\omega_{0}$ [25]. The entanglement in the transmitted light is a consequence of the collective nonlinear response of the atomic ensemble. Entangled photons from each atom constructively interfere which leads to collectively enhanced squeezing. At the single atom level, the entanglement arises from the two-photon scattering process of resonance fluorescence, as depicted in Fig. 1(a). Two resonant photons that arrive during the lifetime of the excited state are scattered into spectrally entangled blue- and red-detuned sidebands, resulting in energy-time 


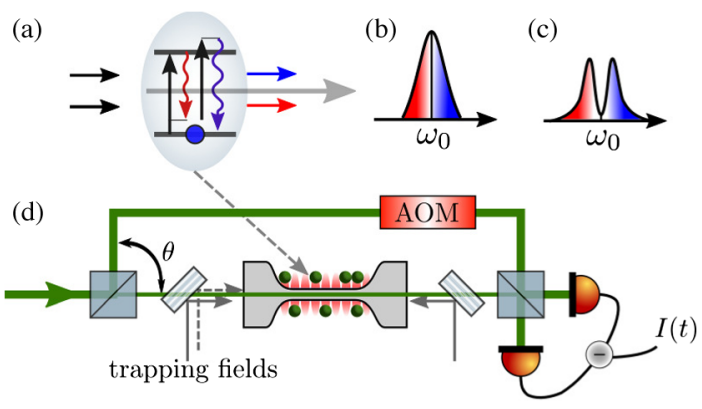

FIG. 1. (a) Atom-mediated photon interaction: two photons interact with a two-level atom and exchange energy such that they become red detuned and blue detuned with respect to the incident light. (b) The scattered light exhibits a Lorentzian shaped spectrum. (c) As the generated photon pairs propagate through the ensemble, absorption around the atomic resonance $\omega_{0}$ attenuates the central part of the spectrum, leaving red- and blue-detuned sidebands. (d) Schematic setup: probe light exiting the nanofiber interferes with a local oscillator at phase $\theta$ on a 50:50 beam splitter and is analyzed on balanced photodetectors. Atoms are trapped in the evanescent field of the nanofiber waist of a tapered optical fiber by a combination of red-detuned standing-wave light field at a wavelength of $935 \mathrm{~nm}$ (solid gray line) and blue-detuned running-wave light field (dashed gray line) at $685 \mathrm{~nm}$.

entangled photon pairs. This process resembles degenerate four-wave mixing (FWM) by a Kerr nonlinearity [26]. However, unlike FWM in a single-mode cavity QED [27-29], the multimode nature of the cascaded system produces richer physics and requires a full multimode treatment of the light field. In our theoretical and experimental analysis we characterize the photon pairs by measuring the resulting quadrature squeezing. The latter allows us to infer the magnitude and the phase of the entangled part of the two-photon wave function.

We consider the physical setting of $N$ emitters weakly coupled to a single-mode continuum of the electromagnetic field with a continuous spectrum. In our setup, we use lasercooled cesium atoms in the evanescent field surrounding a tapered optical fiber with a $400 \mathrm{~nm}$ diameter. The atoms are trapped in two 1D arrays of trapping minima along the nanofiber created through a combination of red- and bluedetuned fiber guided light fields [30-32]. The atoms are located at a distance of $\sim 250 \mathrm{~nm}$ from the fiber surface, and each site contains at most one atom. The nanofiber-guided probe field of power $P_{\text {in }}$ is near-resonant with the cesium D2-line transition and interfaces the atoms via the evanescent field of the nanofiber mode as depicted in Fig. 1(d) [33]. The coupling of individual atoms to the nanofiber mode is weak, with a coupling constant $\beta=\Gamma_{\mathrm{wg}} / \Gamma_{\text {tot }}=$ $0.0070(5)$ [34], where $\Gamma_{\mathrm{wg}}$ is the spontaneous emission rate into the waveguide and $\Gamma_{\text {tot }}=2 \pi \times 5.2 \mathrm{MHz}$ is the total emission rate. We analyze the transmitted light via a balanced homodyne detection scheme: The output is first filtered from the trapping light fields and then sent to a
50:50 beam splitter where it is mixed with a local oscillator (LO) field [38-40] as shown in Fig. 1(d). The two outputs are measured on balanced photodetectors, and we record the amplified differential current $I(t)$ between both photodiodes, which is proportional to the field quadrature $X_{\theta}(t)$. Here, $\theta$ is the relative phase between the LO and the probe field. The quadrature operator is given by $\hat{X}_{\theta}(t)=\frac{1}{2}\left[\hat{a}(t) e^{i \theta}+\hat{a}^{\dagger}(t) e^{-i \theta}\right]$, where $\hat{a}\left(\hat{a}^{\dagger}\right)$ is the annihilation (creation) operator. For a classical (coherent) state of light $\left\langle\hat{X}_{\theta}(t)\right\rangle$ oscillates as $\cos (\theta)$, and the fluctuations $\Delta \hat{X}_{\theta}(t)=\hat{X}_{\theta}(t)-\left\langle\hat{X}_{\theta}(t)\right\rangle$ are independent of the phase $\theta$ and have minimum uncertainty, i.e., they are at the shot noise limit.

We probe an ensemble of $N$ atoms with an incident light that has a saturation parameter $s=P_{\text {in }} / P_{\text {sat }}$, where $P_{\text {sat }}=\hbar \omega \Gamma_{\text {tot }} /(8 \beta)=136 \pm 10 \mathrm{pW}$, and detuning $\Delta$ from the atomic resonance. The transmitted light experiences a change in phase and amplitude upon interaction such that the output field has the average field quadrature

$$
\left\langle\hat{X}_{\theta}(t)\right\rangle=\left|t_{\Delta}\right|^{N} s^{1 / 2} \sqrt{\frac{\Gamma_{\text {tot }}}{8 \beta}} \cos (\theta+\delta)+\mathcal{O}(s) .
$$

Here, $\mathcal{O}(s)$ refers to terms of order $s$ and higher, and $\delta=\operatorname{Arg}\left\{t_{\Delta}^{N}\right\}$, where $t_{\Delta}=1-2 \beta /\left(1-2 i \Delta / \Gamma_{\text {tot }}\right)$ is the single-photon transmission coefficient of a single atom. The atoms also modify the variance $\left\langle\Delta \hat{X}_{\theta}^{2}(t)\right\rangle$ and lead to quadrature squeezing. For squeezed light, the quadrature variance is smaller than the shot noise level for a certain value of $\theta$. More precisely, the variance $\left\langle\Delta \hat{X}_{\theta}^{2}(t)\right\rangle$ oscillates twice as fast as $\left\langle\hat{X}_{\theta}(t)\right\rangle$ and exhibits a $\cos (2 \theta+\varphi)$ modulation. For a resonant drive the phase offset $\varphi$ is zero and the light is amplitude squeezed with maximum squeezing observed for $\theta=(0, \pi)$.

In our setup, the squeezing is induced by the nonlinear optical response of atoms which behave as effective twolevel systems. In the following, we limit our discussion to powers well below saturation $s / 8 \ll 1$ such that we can neglect events where three or more photons arrive simultaneously at any atom. The transmitted part of the twophoton wave function can be expressed in terms of separable and entangled photons. In the frame rotating with $\omega_{0}$, the two-photon wave function is

$$
\psi_{2}\left(x_{1}, x_{2}\right)=t_{\Delta}^{2 N}-\phi_{N}\left(x_{1}-x_{2}\right) .
$$

The first term denotes separable photons which are monochromatic traveling waves extended in space. Upon interaction with each emitter, each of the separable photons acquires a transmission coefficient $t_{\Delta}$. The entangled part of the wave function is not separable and is a localized function of the relative coordinate $x_{1}-x_{2}$. On resonance $(\Delta=0)$ and for a single emitter it is a decaying exponential 


$$
\phi_{N=1}\left(x=x_{1}-x_{2}\right)=4 \beta^{2} e^{-|x| \Gamma_{\text {tot }} /\left(2 v_{g}\right)},
$$

where $v_{g}$ is the group velocity of the photons. For $N$ emitters the entangled part of the wave function $\phi_{N}(x)$ was computed in Ref. [17].

Introducing the normally ordered squeezing spectrum

$$
S_{\theta}(\omega)=\int_{-\infty}^{\infty}\left\langle: \Delta \hat{X}_{\theta}(\tau) \Delta \hat{X}_{\theta}(0):\right\rangle e^{i \omega \tau} d \tau
$$

allows relating the entangled part of the two-photon wave function with the variance of the field quadrature (see Supplemental Material [34] for detailed calculations). Here, $\therefore$ : denotes normal ordering and the normally ordered squeezing spectrum of a coherent state yields $S_{\theta}(\omega)=0$, while squeezed light yields $S_{\theta}(\omega)<0$. For the states generated in our experiment and in the case of weak saturation, the squeezing spectrum $S_{\theta}(\omega)$ and the spectrum of the entangled photons $\phi_{N}(\omega)=\int \phi_{N}(x) e^{-i \omega x / v_{g}} d x$ are linked by

$S_{\theta}(\omega)=-\frac{\Gamma_{\text {tot }}}{16 \beta}\left|\phi_{N}(\omega)\right| \cos \left[2 \theta+\varphi_{N}(\omega)\right] s+\mathcal{O}\left(s^{2}\right)$,

where we introduced the phase and the magnitude of the spectrum of the entangled two-photon wave function as $\phi_{N}(\omega)=\left|\phi_{N}(\omega)\right| e^{i \varphi_{N}(\omega)}$. In the following, we will first focus on a resonant probe field for which $\phi_{N}(\omega)$ is a real quantity $\left[\varphi_{N}(\omega)=0\right]$. For a single emitter $\phi_{N=1}(\omega)$ is the Fourier transform of Eq. (3) which gives a Lorentzian. Consequently, also the squeezing spectrum has a Lorentzian shape [41]. For $N$ emitters with $N \beta \ll 1$, reabsorption can be neglected and the scattered components constructively interfere since the process relies on forward scattering. A coherent build up of the squeezed photons takes place, and the squeezing spectrum is coherently enhanced, i.e., its amplitude is $N$ times larger than the single atom squeezing spectrum [42]. For large optical depth (OD), the probability that the squeezed photons are scattered again and thereby most likely removed from the fiber cannot be neglected anymore, and the problem becomes a quantum many-body problem. Recently, it has been shown that this problem can be solved exactly up to two-photon input states for chiral coupling where atoms couple only to one propagation direction of the mode [17]. Applying this formalism (see Supplemental Material [34] for detailed calculations) allows calculating the squeezing spectrum for arbitrary $N$. The results from those calculations can be understood in the following manner: photon losses occur predominantly close to the emitters resonance which reduces the observed squeezing close to resonance. For many emitters this leads to a squeezing spectrum which develops sidebands due to the loss of squeezed photons that are resonant with the atoms as we will experimentally show later below.
We typically load a few hundred atoms into the evanescent field trap and determine the number of atoms $N$ in a separate transmission measurement. We then probe the atoms on the cycling transition of the $D 2$ line. The probing lasts for $10-100 \mu \mathrm{s}$, such that heating due to resonant scattering of the probe is small, and the number of trapped atoms does not change significantly during probing. After the homodyne measurement, we eject the atoms from the trap, shift the LO field frequency by $1 \mathrm{MHz}$, and increase its power in order to observe a beat note between the probe field and the LO. From the beat note, we extract the relative phase $\theta$ between the probe field and the local oscillator at the moment of the homodyne measurement. After this heterodyning stage, we switch off the probe field and record a vacuum reference where only the LO field is incident on the homodyne detector. We repeat the measurement 10000-100 000 times depending on the dataset and record $I(t)$ in each run. From each experiment cycle, we extract the power spectrum of $I(t)$ during atom probing, the vacuum reference, and the relative phase $\theta$.

In a first step, we extract the noise $\left\langle\Delta I^{2}(\theta)\right\rangle$ and normalize it to the vacuum reference as shown in Fig. 2. We average the noise within the relevant frequency range of $f_{\min }=1.5 \mathrm{MHz}$ and $f_{\max }=23 \mathrm{MHz}$. The lower boundary is chosen to exclude technical noise and the upper boundary to capture the physically relevant frequency range on the order of a few $\Gamma_{\text {tot }}$. The mean atom number during probing consists of $N=169 \pm 12$ trapped atoms. In all measurements, the number of atoms decreases by less than $20 \%$ during probing, which we infer in a separate transmission measurement. The incident field is resonant and has a saturation parameter of $s=0.51 \pm 0.04$. In Fig. 2 data points with atoms show the expected $-\cos (2 \theta)$ modulation of the noise. Values smaller than 1 show that the light is quadrature squeezed. The maximum observed squeezing within the bandwidth $\Delta f=f_{\max }-f_{\min }$ is $0.65 \% \pm 0.12 \%$. We fit the data with the function

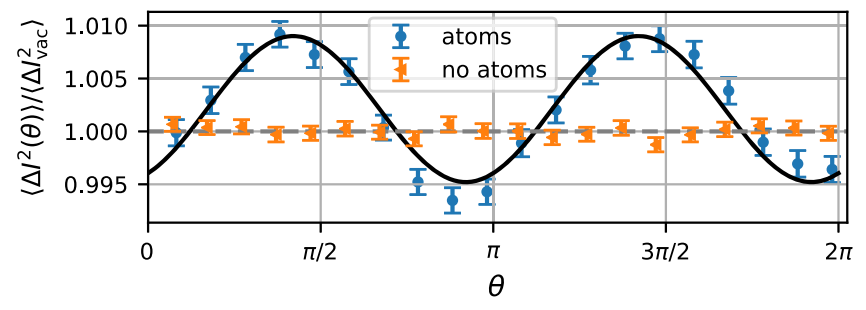

FIG. 2. The observed output noise as a function of the phase $\theta$. The noise is normalized to the vacuum reference and deduced from the frequency range $f_{\text {min }}=1.5 \mathrm{MHz}$ to $f_{\max }=23 \mathrm{MHz}$. The signal obtained with trapped atoms (shown in circles) is compared to the measurement without atoms (shown in triangles). We fit the experimental data with trapped atoms using the function $-A \cos (2 \theta+\varphi)+c$ (black line) which reveals the expected $\cos (2 \theta)$ modulation. Squeezing occurs for $\theta$ around 0 and $\pi$ while antisqueezing occurs around $\pi / 2$ and $3 \pi / 2$. The resonant input power is $P_{\text {in }} / P_{\text {sat }}=0.51 \pm 0.04$. 
$-A \cos (2 \theta+\varphi)+c$ where $A$ is the amplitude of the squeezing, and $c$ accounts for additional noise sources in the experiment. We obtain a small value for the squeezing angle $\varphi / \pi=0.1 \pm 0.03$. This shows that the light is almost purely amplitude squeezed, i.e., the strongest squeezing is observed for $\theta$ close to 0 and $\pi$.

In the next step, we make use of the homodyne detection scheme to access the spectrum of the entangled photons. From the experimental power spectrum normalized to the vacuum spectrum, we deduce the normally ordered squeezing spectrum $S_{\theta}(\omega)$ (see Supplemental Material [34] for detailed calculations). Figures 3(a)-3(c) show the normally ordered squeezing spectrum for different $N$ for the most squeezed $(\theta=0, \pi)$ in orange (bright) and the most antisqueezed $(\theta=\pi / 2,3 \pi / 2)$ in blue (dark). Here, we average over a $\theta$ range of $\pm 18^{\circ}$ around both maxima and minima of the noise $\left\langle\Delta I^{2}(\theta)\right\rangle$. At $\theta=(0, \pi)$, for all atom numbers, the squeezing spectrum $S_{\theta}(\omega)$ exhibits fluctuations below zero which confirms that the spectral components created by the two-photon scattering are energy-time entangled [25]. We attribute the deviations at low frequencies mainly to technical low-frequency noise.

In order to determine the spectrum of the entangled twophoton components $\left|\phi_{N}(\omega)\right|$ we make use of the fact that $S_{\theta}(\omega)$ is proportional to $\left|\phi_{N}(\omega)\right|$ and for a resonant probe can be best extracted at the extrema of the cosine modulation $(\theta=0, \pi / 2, \pi, 3 \pi / 2)$. We obtain $\left|\phi_{N}(\omega)\right|$ directly from the squeezing spectra shown in Figs. 3(a)-3(c) by using Eq. (5) and averaging over the absolute value of $S_{\theta}(\omega)$ at the four different values of $\theta$. Panels (d)-(f) in Fig. 3 show the spectrum of the entangled photons $\left|\phi_{N}(\omega)\right|$ for different $N$ from small to large atom numbers. For a small atom number, i.e., a small OD of the atomic ensemble, the spectrum is mainly dominated by a coherent buildup of entangled photons, and the shape of $\phi_{N}(\omega)$ is close to the line shape of the atomic transition as shown in Fig. 3(d). As $N$ is increased, as in (e) and (f), the probability that squeezed photons are scattered a second time and thereby lost from the waveguide mode increases. These events are more likely close to the atomic resonance. Consequently, one observes two sidebands, to the left and to the right of the atomic transition. Already for $N=194 \pm 14$ the spectrum strongly deviates from a Lorentzian. For $N=262 \pm 19$, the entangled photons with frequency components close to resonance have almost vanished and the entangled photon pairs are concentrated in the sidebands.

So far, we considered a resonant probe field which leads to zero phase of the entangled part of the two-photon wave function $\left[\varphi_{N}(\omega)=0\right]$. In the following, we measure the detuning dependence of $\varphi_{N}(\omega)$. We probe $N=140$ atoms with $s=0.37 \pm 0.03$ for different detunings $\Delta$. First, we focus on the phase of the squeezing, i.e., the phase of the entangled two-photon wave function $\varphi_{N}(\tau)=\operatorname{Arg}\left\{\phi_{N}(\tau)\right\}$ averaged over $\Delta f$. As in Fig. 2, we fit the averaged noise and extract the offset angle $\varphi$. Since the bandwidth $\Delta f$ contains the relevant frequencies, this method is equivalent to an integration over all frequencies and we introduce the frequency integrated entangled two-photon wave function $2 \pi \phi_{N}(\tau=0)=\int_{-\infty}^{\infty} \phi_{N}(\omega) d \omega$. Figure $4(\mathrm{a})$ shows the experimental values for $\varphi_{N}(\tau=0)$ together with its theoretical prediction.

In order to reconstruct the complex-valued function $\phi_{N}(\omega)=\left|\phi_{N}(\omega)\right| e^{i \varphi_{N}(\omega)}$, we access $\varphi_{N}(\omega)$ by fitting the phase in each frequency range individually. Figure 4(b) shows the phase of $\phi_{N}(\omega)$ as a function of $\omega$ together with
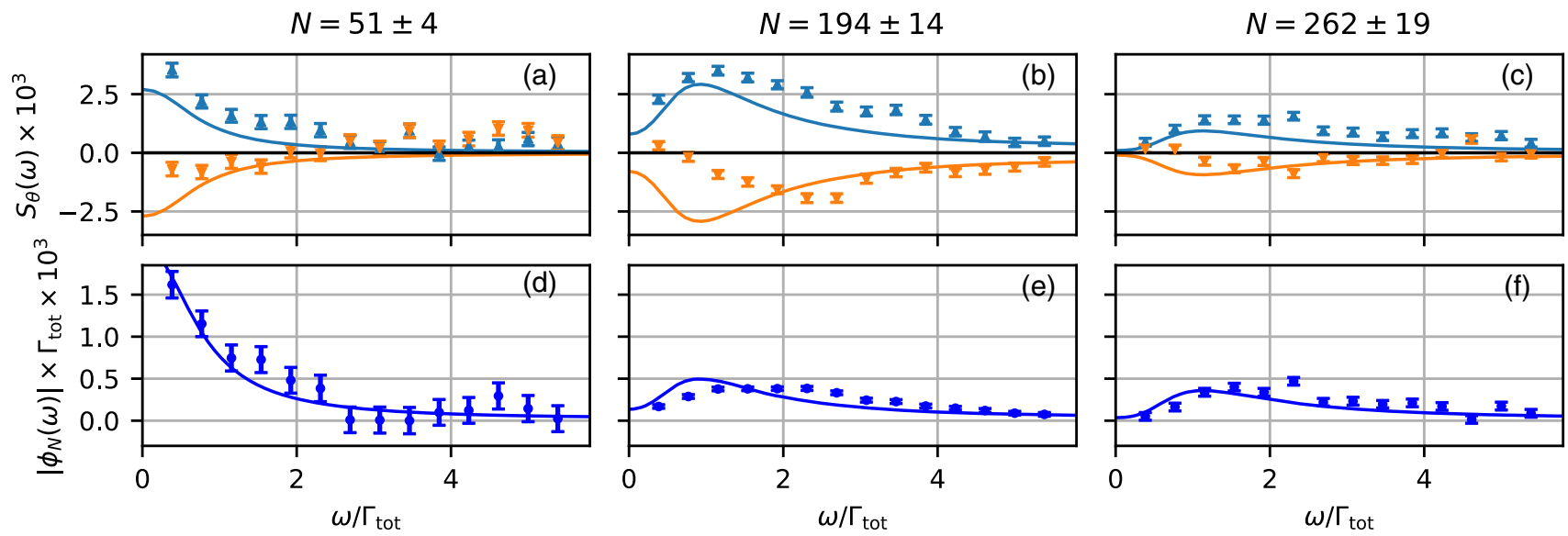

FIG. 3. The first row of panels, (a)-(c), shows the squeezing spectrum $S_{\theta}(\omega)$ for three different atom numbers at input powers $s=(0.15 \pm 0.01,0.67 \pm 0.05,0.29 \pm 0.02)$ (from left to right) at the angle of largest squeezing, $\theta=(0, \pi)$ in orange (bright), and largest antisqueezing, $\theta=(\pi / 2,3 \pi / 2)$ in blue (dark). The corresponding theoretical predictions are shown as solid lines. The second row of panels, (d)-(f) shows the entangled photon spectrum $\left|\phi_{N}(\omega)\right|$ which is deduced from the upper row with the corresponding theoretical prediction (solid line). All theoretical curves are predictions based on the independently measured parameters $\beta$ and $N$, without any free fit parameter taking into account the effect of independently estimated photon loss and detection efficiency in our setup $[34,43,44]$. 

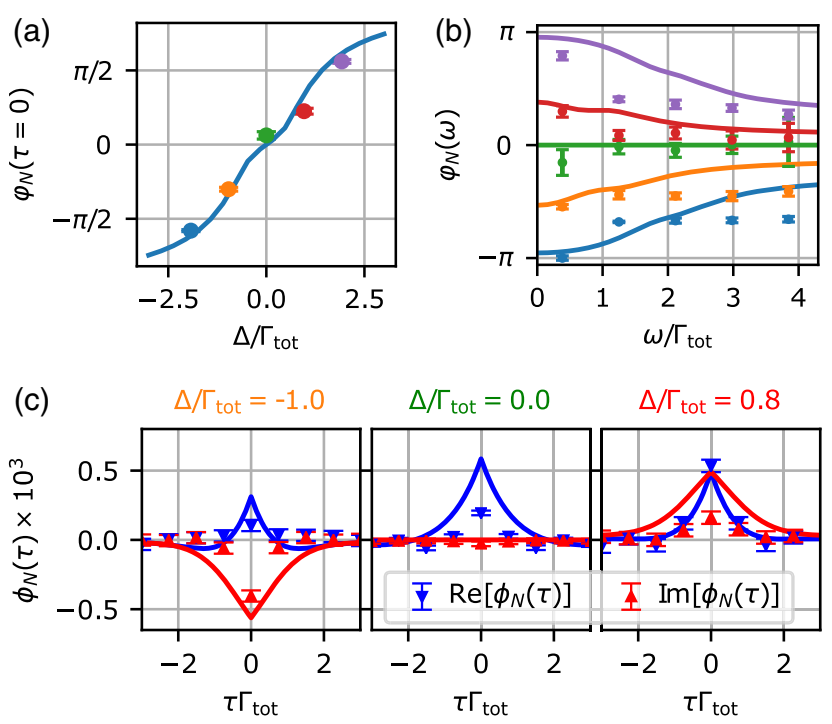

FIG. 4. The phase of the entangled part of the two-photon wave function $\varphi_{N}$ for $N=140$ together with the corresponding theoretical predictions in solid lines. (a) The integrated phase $\varphi_{N}(\tau=0)=\operatorname{Arg}\left\{\phi_{N}(\tau=0)\right\}$ for different detunings $\Delta$. (b) The phase $\varphi(\omega)$ as a function of frequency. The detunings from top to bottom are $\Delta / \Gamma_{\text {tot }}=1.9,0.8,0,-1,-1.9$ and share the same color code as in (a). (c) The reconstructed real and imaginary part of the entangled two-photon time-domain wave function $\phi_{N}(\tau)$. The solid lines show the corresponding theoretical predictions based on the independently measured values of $\beta$ and $N$, without any free fit parameter.

its theoretical prediction for different laser-atom detunings $\Delta$. From the phase and the magnitude in frequency space, one can equivalently obtain the complex time-domain wave function $\phi_{N}(\tau)$ by an inverse Fourier transform. Using this method, we obtain the experimental $\phi_{N}(\tau)$, and Fig. 4(c) shows three examples together with the corresponding theoretical prediction which does not contain any fit parameter.

In conclusion, we observed the generation of squeezed light by sending weak coherent laser light through an ensemble of atoms weakly coupled to a nanofiber. The squeezing spectrum obtained via homodyne measurement gives direct access to the relative phase and magnitude of the two-photon wave function at the fiber output, allowing us to reconstruct the time-dependent two-photon wave function of the transmitted light. These measurements reveal the change in phase and magnitude of the entangled photons for different detunings and relate these to the squeezing spectrum.

A recent theoretical proposal has suggested that correlation measurements can be used to reconstruct the scattering matrix of an arbitrary quantum scatterer [45], and a first experimental step has been taken for a single quantum emitter [46]. While we have focused on reconstructing the two-photon wave function, our measurement could also be extended to reconstruct the entire scattering matrix of an atomic ensemble. Finally, while in this work we have focused on squeezing measurements, which quantify second-order amplitude correlations, studying higher-order correlations is also possible with our approach. For example, a nonzero third-order moment can unveil the expected non-Gaussian nature of the output photons and can also uncover the existence of three-body entanglement.

We are grateful to A. Sørensen, L. Orozco, P. Solano, J.-H. Mueller, B. Hacker, and M. Kraft for stimulating discussions and helpful comments. We thank S. Rind for his support in building the experiment. We acknowledge financial support by the Alexander von Humboldt Foundation, the European Commission under the projects ErBeStA (No. 800942) and the ERC grant NanoQuaNt, and by the Austrian Science Fund (DK CoQuS Project No. W 1210-N16). M. S. acknowledges support by the European Commission (Marie Skłodowska-Curie IF Grant No. 896957). S. M. and K. H. acknowledge funding from DFG through CRC 1227 DQ-mat, projects A05 and A06, and "Niedersächsisches Vorab" through the "Quantum-and Nano-Metrology (QUANOMET)."

*sahand.mahmoodian@sydney.edu.au

'Present address: Department of Electrical Engineering, Columbia University, New York, New York 10027, USA. ${ }^{\ddagger}$ Present address: Centre for Engineered Quantum Systems, School of Physics, The University of Sydney, Sydney New South Wales 2006, Australia.

${ }^{\S}$ maximilian.schemmer@hu-berlin.de

[1] K. M. Birnbaum, A. Boca, R. Miller, A. D. Boozer, T. E. Northup, and H. J. Kimble, Nature (London) 436, 87 (2005).

[2] B. Dayan, A. S. Parkins, T. Aoki, E. P. Ostby, K. J. Vahala, and H. J. Kimble, Science 319, 1062 (2008).

[3] A. Faraon, I. Fushman, D. Englund, N. Stoltz, P. Petroff, and J. Vučković, Nat. Phys. 4, 859 (2008).

[4] A. Reinhard, T. Volz, M. Winger, A. Badolato, K. J. Hennessy, E. L. Hu, and A. Imamoğlu, Nat. Photonics 6, 93 (2012).

[5] A. Reiserer, N. Kalb, G. Rempe, and S. Ritter, Nature (London) 508, 237 (2014).

[6] J. Volz, M. Scheucher, C. Junge, and A. Rauschenbeutel, Nat. Photonics 8, 965 (2014).

[7] C. Hamsen, K. N. Tolazzi, T. Wilk, and G. Rempe, Phys. Rev. Lett. 118, 133604 (2017).

[8] A. M. Guerrero, P. Nussenzveig, M. Martinelli, A. M. Marino, and H. M. Florez, Phys. Rev. Lett. 125, 083601 (2020).

[9] T. Peyronel, O. Firstenberg, Q.-Y. Liang, S. Hofferberth, A. V. Gorshkov, T. Pohl, M. D. Lukin, and V. Vuletić, Nature (London) 488, 57 (2012).

[10] Y. O. Dudin and A. Kuzmich, Science 336, 887 (2012).

[11] V. Parigi, E. Bimbard, J. Stanojevic, A. J. Hilliard, F. Nogrette, R. Tualle-Brouri, A. Ourjoumtsev, and P. Grangier, Phys. Rev. Lett. 109, 233602 (2012). 
[12] D. Maxwell, D. J. Szwer, D. Paredes-Barato, H. Busche, J. D. Pritchard, A. Gauguet, K. J. Weatherill, M. P. A. Jones, and C. S. Adams, Phys. Rev. Lett. 110, 103001 (2013).

[13] S. Baur, D. Tiarks, G. Rempe, and S. Dürr, Phys. Rev. Lett. 112, 073901 (2014).

[14] J. D. Thompson, T. L. Nicholson, Q.-Y. Liang, S. H. Cantu, A. V. Venkatramani, S. Choi, I. A. Fedorov, D. Viscor, T. Pohl, M. D. Lukin, and V. Vuletić, Nature (London) 542, 206 (2017).

[15] N. Stiesdal, J. Kumlin, K. Kleinbeck, P. Lunt, C. Braun, A. Paris-Mandoki, C. Tresp, H. P. Büchler, and S. Hofferberth, Phys. Rev. Lett. 121, 103601 (2018).

[16] D. Tiarks, S. Schmidt-Eberle, T. Stolz, G. Rempe, and S. Dürr, Nat. Phys. 15, 124 (2019).

[17] S. Mahmoodian, M. Čepulkovskis, S. Das, P. Lodahl, K. Hammerer, and A.S. Sørensen, Phys. Rev. Lett. 121, 143601 (2018).

[18] A. S. Prasad, J. Hinney, S. Mahmoodian, K. Hammerer, S. Rind, P. Schneeweiss, A. S. Sørensen, J. Volz, and A. Rauschenbeutel, Nat. Photonics 14, 719 (2020).

[19] L. Hanschke, L. Schweickert, J. C. López Carreño, E. Schöll, K. D. Zeuner, T. Lettner, E. Z. Casalengua, M. Reindl, S. F. C. da Silva, R. Trotta, J. J. Finley, A. Rastelli, E. del Valle, F. P. Laussy, V. Zwiller, K. Müller, and K. D. Jöns, Phys. Rev. Lett. 125, 170402 (2020).

[20] R. E. Slusher, L. W. Hollberg, B. Yurke, J. C. Mertz, and J. F. Valley, Phys. Rev. Lett. 55, 2409 (1985).

[21] Z. H. Lu, S. Bali, and J. E. Thomas, Phys. Rev. Lett. 81, 3635 (1998).

[22] A. Ourjoumtsev, A. Kubanek, M. Koch, C. Sames, P. W. H. Pinkse, G. Rempe, and K. Murr, Nature (London) 474, 623 (2011).

[23] C. H. H. Schulte, J. Hansom, A. E. Jones, C. Matthiesen, C. Le Gall, and M. Atatüre, Nature (London) 525, 222 (2015).

[24] D. F. Walls and P. Zoller, Phys. Rev. Lett. 47, 709 (1981).

[25] S. Zippilli, G. D. Giuseppe, and D. Vitali, New J. Phys. 17, 043025 (2015).

[26] R. L. Carman, R. Y. Chiao, and P. L. Kelley, Phys. Rev. Lett. 17, 1281 (1966).

[27] J. E. Reiner, W. P. Smith, L. A. Orozco, H. J. Carmichael, and P. R. Rice, J. Opt. Soc. Am. B 18, 1911 (2001).

[28] M. D. Reid and D. F. Walls, Phys. Rev. A 34, 4929 (1986).

[29] G. V. Varada, M. Sunjay Kumar, and G. S. Agarwal, Opt. Commun. 62, 328 (1987).

[30] F. Le Kien, V. I. Balykin, and K. Hakuta, Phys. Rev. A 70, 063403 (2004).
[31] E. Vetsch, D. Reitz, G. Sagué, R. Schmidt, S. T. Dawkins, and A. Rauschenbeutel, Phys. Rev. Lett. 104, 203603 (2010).

[32] N. V. Corzo, B. Gouraud, A. Chandra, A. Goban, A. S. Sheremet, D. V. Kupriyanov, and J. Laurat, Phys. Rev. Lett. 117, 133603 (2016).

[33] E. Vetsch, S. T. Dawkins, R. Mitsch, D. Reitz, P. Schneeweiss, and A. Rauschenbeutel, IEEE J. Sel. Top. Quantum Electron. 18, 1763 (2012).

[34] See Supplemental Material at http://link.aps.org/supplemental/ 10.1103/PhysRevLett.127.123602 for the determination of $\beta$, a detailed discussion of the detection efficiency, and detailed calculations of the squeezing spectrum $S_{\theta}(\omega)$, which includes Refs. [35-37].

[35] S. Stenholm, Foundations of Laser Spectroscopy (Dover Publications, Mineola, 2012).

[36] A. I. Lvovsky, in Photonics: Scientific Foundations, Technology and Applications, Volume 1 (Wiley, New York, 2015), pp, 121-163, https://onlinelibrary.wiley.com/doi/ abs/10.1002/9781119009719.ch5.

[37] R. Mitsch, C. Sayrin, B. Albrecht, P. Schneeweiss, and A. Rauschenbeutel, Phys. Rev. A 89, 063829 (2014).

[38] M. Das, A. Shirasaki, K. P. Nayak, M. Morinaga, F. L. Kien, and K. Hakuta, Opt. Express 18, 17154 (2010).

[39] J.-B. Béguin, E. M. Bookjans, S. L. Christensen, H. L. Sørensen, J. H. Müller, E. S. Polzik, and J. Appel, Phys. Rev. Lett. 113, 263603 (2014).

[40] S. Jalnapurkar, P. Anderson, E. S. Moiseev, P. Palittapongarnpim, A. Narayanan, P. E. Barclay, and A. I. Lvovsky, Opt. Lett. 44, 1678 (2019).

[41] M. J. Collett, D. F. Walls, and P. Zoller, Opt. Commun. 52, 145 (1984).

[42] A. Heidmann and S. Reynaud, J. Phys. 46, 1937 (1985).

[43] J. Appel, D. Hoffman, E. Figueroa, and A. I. Lvovsky, Phys. Rev. A 75, 035802 (2007).

[44] H.-A. Bachor and T.C. Ralph, Quantum noise: Basic measurements and techniques, in A Guide to Experiments in Quantum Optics (John Wiley \& Sons, Ltd, 2019), Chap. 8, pp. 269-301.

[45] T. Ramos and J. J. García-Ripoll, Phys. Rev. Lett. 119, 153601 (2017).

[46] H. L. Jeannic, T. Ramos, S. F. Simonsen, T. Pregnolato, Z. Liu, R. Schott, A. D. Wieck, A. Ludwig, N. Rotenberg, J. J. García-Ripoll, and P. Lodahl, Phys. Rev. Lett. 126, 023603 (2021). 